\title{
Life-threatening tension pneumothorax after unsuccessful tracheostomy tube exchange in a trauma patient - A case report -
}

Received June 5, 2019

Revised 1st, July 12, 2019 2nd, July 27, 2019 Accepted July 27, 2019

\section{Corresponding author}

Jeong Heon Park, M.D.

Department of Anesthesiology and Pain Medicine, Dankook University Hospital, 201 Manghyang-ro, Dongnam-gu, Cheonan 31116, Korea

Tel: 82-41-550-6814

Fax: 82-41-550-6814

E-mail: mdpjh@hanmail.net ORCID

https://orcid.org/0000-0003-3852-5390

\section{Min A Kwon, Chaemin Cho, and Jeong Heon Park}

Department of Anesthesiology and Pain Medicine, Dankook University Hospital, Cheonan, Korea

Background: Tracheostomy tube exchange is a common and safe procedure. However, when the tracheocutaneous tract is not completely mature, cannula exchange or endotracheal tube insertion via the tracheostomy site can rarely induce life-threatening complications, including subcutaneous emphysema, loss of airway, tension pneumothorax, and pneumoperitoneum.

Case: We report a case of life-threatening tension pneumothorax developed during tracheostomy tube exchange with a reinforced endotracheal tube for a planned facial surgery after recent tracheostomy in a trauma patient.

Conclusions: Understanding of the pathogenesis and the use of preventive strategies based on it are expected to provide safer and more effective anesthetic management to patients with tracheostomy.

Keywords: Pneumothorax; Subcutaneous emphysema; Tracheostomy.
Tracheostomy tube exchange, which is commonly used in the intensive care unit (ICU) or operating room, is a relatively safe procedure. However, when the tracheocutaneous tract has not fully matured, or the distance between the skin and the trachea is much longer because of obesity, edema, or bleeding, the layers of the surrounding tissues are loose and the possibility of entry in the false lumen increases, which thereby increases the possibility of serious complications [1].

We describe a case of fatal tension pneumothorax due to failed tracheostomy tube exchange during anesthesia for facial surgery and discuss the appropriate preparation and anesthetic management.

\section{CASE REPORT}

Informed consent to publish this case was obtained from the patient. A 48-year-old man was admitted to the emergency room due to a pedestrian traffic accident. He had skull fracture, spontaneous subarachnoid hemorrhage, upper and lower mandibular fractures, ankle fracture, and deep facial lacerations. In the emergency room, his consciousness was stuporous, and oral and nasal bleeding were severe. After emergency blood transfusion, he underwent tracheal intubation and was placed on a ventilator in the ICU. Prolonged ventilator therapy was expected, and tracheostomy was performed with the open surgical technique. Postoperative chest X-ray showed no specific findings (Fig. 1).

Three days after the tracheostomy, the planned surgery for mandibular fracture and facial deep laceration was performed. In the operating room, his consciousness was deep drowsy. The blood pressure was 130/75 $\mathrm{mmHg}$; heart rate was 90 beats/min; oxygen saturation $\left(\mathrm{SpO}_{2}\right)$ was $98 \%$. The

This is an Open Access article distributed under the terms of the Creative Commons Attribution Non-Commercial License (http://creativecommons.org/licenses/by-nc/4.0) which permits unrestricted non-commercial use, distribution, and reproduction in any medium, provided the original work is properly cited. 


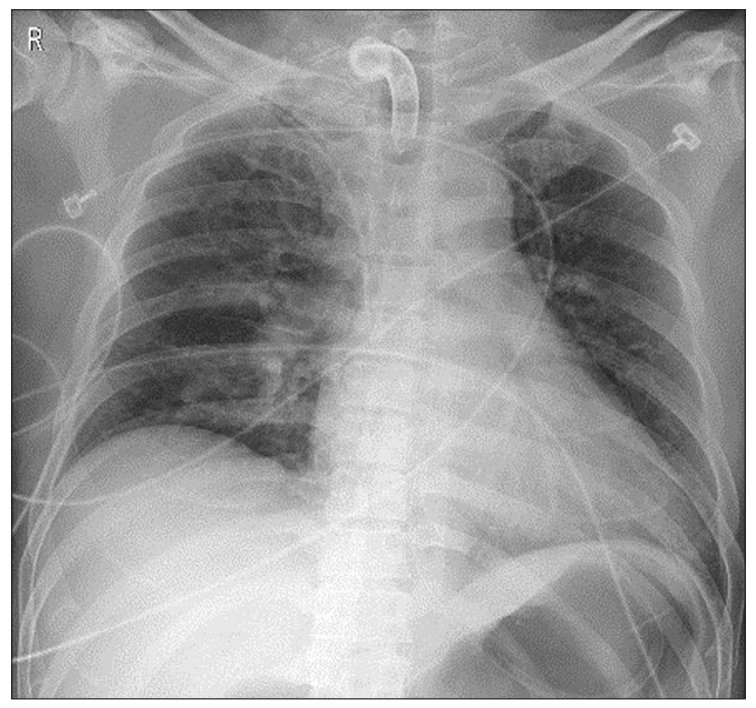

Fig. 1. Preoperative chest $X$-ray.

anesthesiologist placed the reinforced tube instead of the $\mathrm{T}$-cannula for surgical visibility and risk of disconnection. The patient was in the supine position. After tracheostomy suction and preoxygenation, $60 \mathrm{mg}$ of lidocaine, $120 \mathrm{mg}$ of propofol, and $100 \mu \mathrm{g}$ of fentanyl were administered. After confirming that there was no problem with ventilation, $50 \mathrm{mg}$ of rocuronium bromide was administered, followed by sufficient manual ventilation. We removed the T-cannula of 7.0$\mathrm{mm}$ internal diameter and placed a reinforced endotracheal tube of 6.5-mm internal diameter.

However, the endotracheal tube could not be inserted through the tracheostomy site. We attempted a smaller size of reinforced tube insertion several times but were not successful. $\mathrm{SpO}_{2}$ decreased from $95 \%$ to $50 \%$ gradually. We reinserted the T-cannula, but ventilation was not possible. $\mathrm{SpO}_{2}$ dropped below $35 \%$. An emergency call was immediately made to ask help from other anesthesiologists. Ambubagging was continued, but $\mathrm{SpO}_{2}$ did not rise. We applied an arterial catheter to the left radial artery, and blood gas analysis showed: $\mathrm{pH}, 7.2$; partial pressure of oxygen $\left(\mathrm{PaO}_{2}\right), 22$; partial pressure of carbon dioxide $\left(\mathrm{PaCO}_{2}\right), 72$; and oxygen saturation $\left(\mathrm{SaO}_{2}\right), 25 \%$. Breath sounds in the right lung field were not audible, and we felt some crepitus in the right upper chest area. Emergency chest X-ray showed a huge amount of tension pneumothorax in the right lung of the patient (Fig. 2). We requested a thoracic surgeon for chest tube insertion. Simultaneously, we performed emergency thoracentesis in the right thorax using $16-\mathrm{G}$ catheter. After removing the stylet of

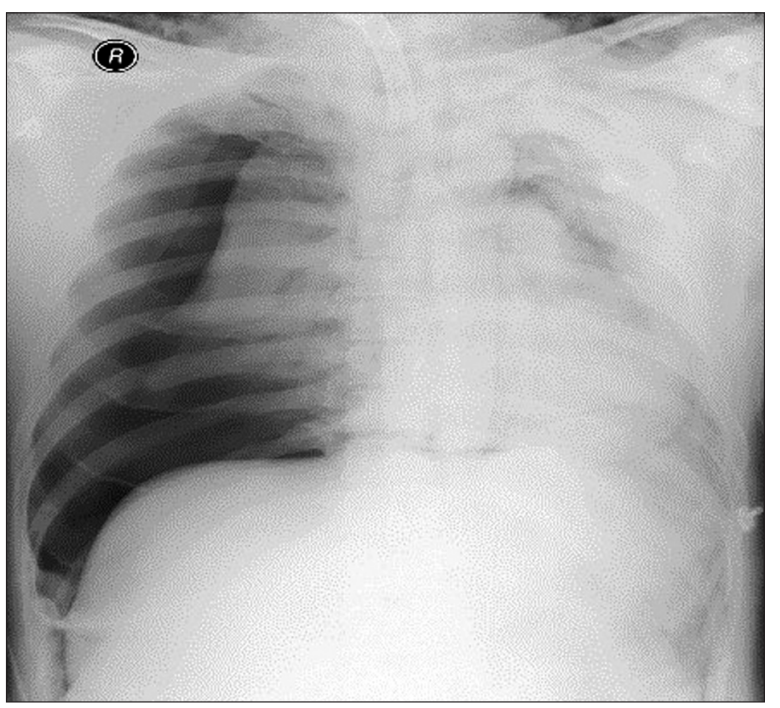

Fig. 2. Chest $\mathrm{X}$-ray after failed tracheostomy tube exchange.

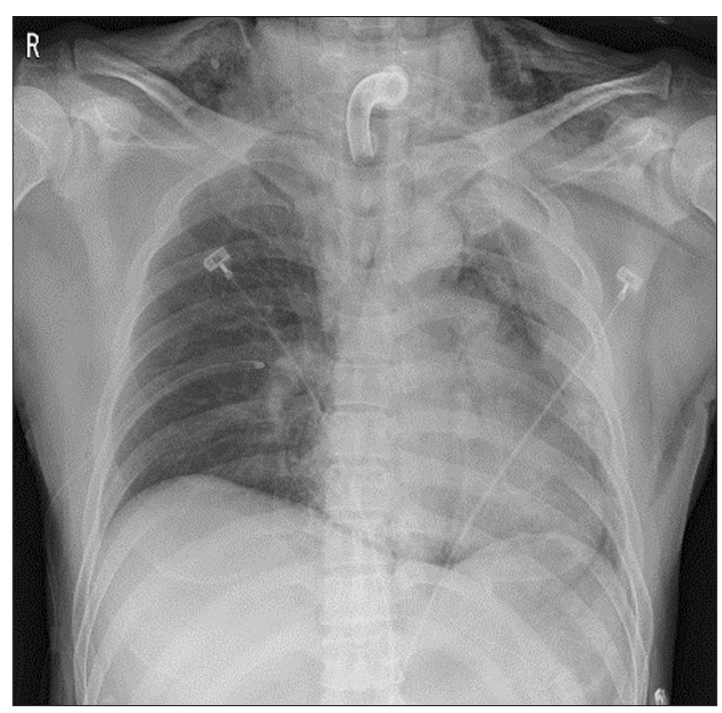

Fig. 3. Chest X-ray after closed thoracotomy.

the catheter, we heard the sound of air gushing out. Ventilation was slightly smoothed in one minute after insertion, and $\mathrm{SpO}_{2}$ slightly increased. Five minutes after catheterization, closed thoracotomy was performed by the thoracic surgeon, and $\mathrm{SpO}_{2}$ rapidly increased to $70-80 \%$. Oxygen saturation remained above $95 \%$, five minutes after the chest tube insertion, and was normalized after $25 \mathrm{~min}$. The arterial gas analysis showed: $\mathrm{pH}, 7.33 ; \mathrm{PaO}_{2}, 105 ; \mathrm{PaCO}_{2}, 45$; and $\mathrm{SaO}_{2}, 98 \%$. The chest radiographs showed that the collapsed lung had re-expanded without pneumothorax (Fig. 3). An Ear Nose Throat (ENT) surgeon repositioned the T-cannula. Intraoperative bronchoscopy was performed, but there was no direct 
tracheal injury. The operation was canceled, and the patient was transferred to the ICU, where the lungs were ventilated with an $\mathrm{FiO}_{2}$ of 0.6 , and $\mathrm{SpO}_{2}$ was $97 \%$. Accurate bronchoscopy was performed again, and no significant damage was found in the trachea wall.

The chest tube was removed four days after the closed thoracotomy. Seven days after the thoracotomy, the surgery for mandibular fracture was performed again, without replacing the T-cannula. During anesthesia, $\mathrm{SpO}_{2}$ did not decrease, and vital signs were stable. The operation time was over seven hours, but there were no special perioperative events, especially airway problems. Decannulation was conducted 48 days after the tracheostomy, without any late complications. Two days after decannulation, the patient was discharged with maintaineed tracheostomy opening. One month later, he visited the outpatient clinic with no neurological or respiratory problems.

\section{DISCUSSION}

We report a case of fatal tension pneumothorax developed during tracheostomy tube exchange with a reinforced endotracheal tube in a trauma patient.

Tracheostomy is a common procedure performed in critically ill patients with prolonged ventilator therapy. In addition to conventional open surgical tracheostomy, percutaneous dilation tracheostomy is widely used [2]. Mortality and morbidity were comparable, but percutaneous tracheostomy is easy to perform at bedside in the ICU, saves operating room costs, reduces the risk in serious patients, and has various advantages, such as avoiding infection, wound healing properties, and esthetics [2]. However, in Korea, open surgical tracheostomy is preferred because of the lower economic burden of surgery and safety of cannula insertion.

Tracheostomy is considered a safe procedure, but the incidence of complications varies from $10 \%$ to $20 \%[2,3]$. Serious complications, such as bleeding, tracheal wall damage, pneumothorax, mediastinitis, and death, occur in $0.7-6 \%$ [2]. In particular, patients with morbid obesity have a longer procedure time and higher mortality (6\%) and morbidity (25\% of the loss of airway) [4]. Moreover, the standard tracheostomy tube is relatively short for patients with a long subcutaneous distance; therefore, there is a higher risk of dislodgement.

Tracheostomy-related complications are classified as in- traprocedural (immediately after tracheostomy), early postprocedural ( $\leq 7$ days, before the tract maturation), and late postprocedural ( $>7$ days, after the tract maturation) complications. One-third of the fatal outcomes occur during the procedure and half of the total (49.3\%) lethal complications occur in seven days after the procedure [5]. Bleeding, loss of airway, tracheal perforation, and tension pneumothorax are serious early complications. In late complications, tracheal stenosis is the most common.

Tension pneumothorax is a rare but life-threatening early complication that requires rapid treatment. It usually has a direct tracheal wall injury but can occur without a specific tracheal injury. Fikkers et al. [6] reported that the cause of tension pneumothorax after tracheostomy can be classified into two types: a direct tracheal injury and paratracheal wrong passage to the false lumen without definite tracheal injury [6]. To compensate for hypoxia, vigorously ventilated forced air through the injured trachea makes a considerable amount of pneumomediastinum. It rapidly spreads in the subcutaneous tissue, mediastinum, and pleura of the neck and thorax through the weakest point of the tissues. Subcutaneous emphysema, pneumomediastinum, and pneumothorax can easily develop. Rarely, it may spread to the abdominal cavity along with the hiatal or esophagus sleeve, resulting in incidental pneumoperitoneum without bowel injury [6].

Although there is no obvious tracheal injury, air inflow in the pseudo tract also produces a significant amount of subcutaneous emphysema, which similarly induces a large amount of air to enter the surrounding mediastinum or pleura. Thus, without tracheal damage, it is possible to develop severe complications, such as tension pneumothorax, bilateral pneumothorax, and pneumoperitoneum [6].

In addition to the tracheal injuries, direct pleural damage or alveolar bleb rupture may occur, often in children with a higher position of the pleural dome [2]. Similarly, in adults, the pleural space is also posterolaterally extended up to the level of the tracheostomy site and the distance between the trachea and the pleural border is very close $(5 \mathrm{~mm}$ in cadaver), which can easily cause pleural space injury with posterior wall damage during tracheostomy [7].

Considering the pathogenesis of pneumothorax, the most important aspect of tracheostomy is avoiding direct tracheal injury and not making a false passage to the pseudo tract.

To reduce the risks in blindly performed percutaneous 
tracheostomy, using bronchoscopy is highly recommended. Bronchoscopy helps to identify the location and depth of the cannula and prevent direct injury to the posterior tracheal wall [2].

To prevent the loss of airway, it is common to stitch the trachea and the skin and wrap a securing tape around the neck. However, the first tracheostomy tube exchange is relatively risky because of the possibility of incomplete tract maturation. Maturation of the tracheocutaneous tract generally takes about 2-7 days. Until tract maturation is complete, the layer of surrounding tissues is still loose, and hence, it could enter the pseudo tract. In particular, the risk of loss of airway is much greater if the length of the stoma track is long because of edema, bleeding, or excessive fat tissues [8]. To confirm tract maturation and remove the granulation tissue, the first tracheostomy tube exchange is usually performed by ENT surgeons $48 \mathrm{~h}$ after the tracheostomy. The mean duration was about five days [9]. In our case, the surgery was performed before the first tracheostomy tube exchange. Because tracheostomy tract maturation was not complete, we needed ENT consultation with sufficient preparation for emergencies, such as loss of airway.

For a safe procedure, the patient should be placed in a neck extension posture at an appropriate height under the shoulder for proper visualization, with head raised $15-20^{\circ}$ to reduce venous engorgement [2]. With sufficient preoxygenation, preparation of the intubating device and suctioning system for emergency intubation is essential. After gentle endotracheal suction, the existing T-cannula should be carefully removed and replaced with the same-sized or smaller endotracheal tube or other tracheostomy tube, avoiding entrance in the peritracheal false lumen. If the maturation is incomplete, or there is a risk of bleeding, edema, or obesity, safer methods are recommended using various exchangers, such as Levin tube, suction catheter, bougie, or tube exchanger. Proper positioning of replaced tracheostomy can be confirmed on exhaled $\mathrm{CO}_{2}$ monitoring or good ventilation profile with proper airway pressure [2]. Immediately after the procedure, bronchoscopy may be used to check for tracheal injury. Even when there is no direct tracheal damage, the possibility of tension pneumothorax should be considered when ventilation is not appropriate after tracheostomy tube exchange, desaturation deteriorates, or significant hypotension is present.
Temporary desaturation often occurs during tracheostomy tube exchange. However, when life-threatening hypoxia occurs, as in this case, endotracheal intubation should be attempted promptly, irrespective of the cause of the complication. Mask ventilation is often difficult to achieve because of air leakage through a tracheostomy opening in the tracheal wall. The balloon of the endotracheal tube should be placed under the tracheostomy level, and desaturation should be treated.

When the tension pneumothorax is strongly suspected, immediate chest tube insertion is necessary. When it is not available, large-needle thoracotomy can be performed to relieve tension, as in our case. Chest X-ray soon after the thoracotomy may be not effective to confirm the efficacy of the procedure. It might take longer for re-expansion of the lungs. Tracheostomy tube exchange is a commonly performed, safe procedure but rarely causes life-threatening complications, such as tension pneumothorax. Understanding the pathogenesis and the use of preventive strategies based on this may provide safer and effective anesthetic management in tracheostomy patients.

\section{CONFLICTS OF INTEREST}

No potential conflict of interest relevant to this article was reported.

\section{ORCID}

Min A Kwon, https://orcid.org/0000-0002-7253-3768

Chaemin Cho, https://orcid.org/0000-0003-4723-9353

\section{REFERENCES}

1. Heyrosa MG, Melniczek DM, Rovito P, Nicholas GG. Percutaneous tracheostomy: a safe procedure in the morbidly obese. J Am Coll Surg 2006; 202: 618-22.

2. Engels PT, Bagshaw SM, Meier M, Brindley PG. Tracheostomy: from insertion to decannulation. Can J Surg 2009; 52: 427-33.

3. Fikkers BG, Briedé IS, Verwiel JM, Van Den Hoogen FJ. Percutaneous tracheostomy with the Blue Rhino trade mark technique: presentation of 100 consecutive patients. Anaesthesia 2002; 57: 1094-7.

4. El Solh AA, Jaafar W. A comparative study of the complications of surgical tracheostomy in morbidly obese critically ill patients. 
Crit Care 2007; 11: R3.

5. Simon M, Metschke M, Braune SA, Püschel K, Kluge S. Death after percutaneous dilatational tracheostomy: a systematic review and analysis of risk factors. Crit Care 2013; 17: R258.

6. Fikkers BG, van Veen JA, Kooloos JG, Pickkers P, van den Hoogen FJ, Hillen B, et al. Emphysema and pneumothorax after percutaneous tracheostomy: case reports and an anatomic study. Chest 2004; 125: 1805-14.

7. Escarment J, Suppini A, Sallaberry M, Kaiser E, Cantais E, Palmi- er B, et al. Percutaneous tracheostomy by forceps dilation: report of 162 cases. Anaesthesia 2000; 55: 125-30.

8. Hwang SM, Jang JS, Yoo JI, Kwon HK, Lee SK, Lee JJ, et al. Difficult tracheostomy tube placement in an obese patient with a short neck -a case report-. Korean J Anesthesiol 2011; 60: 434-6.

9. Tabaee A, Lando T, Rickert S, Stewart MG, Kuhel WI. Practice patterns, safety, and rationale for tracheostomy tube changes: a survey of otolaryngology training programs. Laryngoscope 2007; 117: 573-6. 